\title{
The microRNA gene bta-mir-2313 in cattle: an atlas of regulatory elements and an association analysis with growth and carcass traits in the Slovenian Simental cattle breed
}

\author{
Špela Malovrh et al.
}

Correspondence to: Peter Dovč (peter.dovc@bf.uni-lj.si)

The copyright of individual parts of the supplement might differ from the CC BY 4.0 License. 
Supplement Table S1: Genomic location of 17 QTL, overlapping the bta-miR-2313 gene.

\begin{tabular}{|l|l|l|l|l|l|}
\hline $\begin{array}{l}\text { QTL } \\
\text { symbol }\end{array}$ & QTL ID & QTL trait & $\begin{array}{l}\text { QTL peak } \\
(\mathrm{cM})\end{array}$ & $\begin{array}{l}\text { QTL span } \\
(\mathrm{cM})\end{array}$ & $\begin{array}{l}\text { QTL span (bp), } \\
\text { chromosome 15 }\end{array}$ \\
\hline AD & 5122 & Abomasum displacement & 67.8 & $58.3-74.1$ & $28737753-36469420$ \\
\hline BFCI & 1594 & Body form composite index & 65 & $54.286-75.71$ & $31549304-37655364$ \\
\hline FA-C13:0 & 19626 & Tridecylic acid content & 41.81 & $0.00-83.62$ & $20-59060134$ \\
\hline FERIND & 62361 & Fertility index & 48.31 & $47.61-49.00$ & $34177504-35177124$ \\
\hline FTPL & 1595 & Teat placement - front & 65 & $54.286-75.71$ & $31549304-37655364$ \\
\hline KPHCWT & 1335 & $\begin{array}{l}\text { Kidney, pelvic, and heart fat } \\
\text { percentage }\end{array}$ & 45 & $21-69$ & $9532674-36158521$ \\
\hline LIVP & 12195 & Liver percentage & 56 & $39.07-73.63$ & $19145683-36123213$ \\
\hline MARBL & 10999 & Marbling score & 77.94 & $59.27-84.89$ & $29452266-40819540$ \\
\hline MHT & 11001 & Height (mature) & 84.89 & $59.27-104.9$ & $29452266-50231208$ \\
\hline MWT & 10997 & Body weight (mature) & 59.27 & $59.27-77.94$ & $29452266-36441038$ \\
\hline PTAT & 1597 & PTA type & 65 & $54.286-75.71$ & $31549304-37655364$ \\
\hline SF & 20794 & Shear force & 44.90 & $44.02-45.78$ & $31599942-63827753$ \\
\hline THUWDT & 1599 & Thurl width & 65 & $54.286-75.71$ & $31549304-37655364$ \\
\hline UC & 1600 & Udder cleft & 65 & $54.286-75.71$ & $31549304-37655364$ \\
\hline UCI & 1602 & Udder composite index & 65 & $54.286-75.71$ & $31549304-37655364$ \\
\hline WWT & 10996 & Body weight (weaning) & 59.27 & $54.29-77.94$ & $31552250-36441038$ \\
\hline WWT & 10998 & Body weight (weaning) & 59.27 & $54.29-77.94$ & $31552250-36441038$ \\
\hline
\end{tabular}

Supplement Figure S1: Polymorphism rs41761413C>T within mature miRNA bta-miR-2313 seed region causing formation of a novel seed region, annotated to a miRNA gene bta-miR-3600. Polymorphism within the seed region also causes a change in the number of predicted targets (3393/3789).

\begin{tabular}{|l|c|}
\hline $\begin{array}{l}\text { bta-miR-2313-5p } \\
\text { GUGCAGC } \\
\text { GUGCACA } \\
\text { GUGCACC } \\
\text { GUGCAGA }\end{array}$ & $\begin{array}{c}3393 \text { transcripts with } \\
\text { predicted miRNA sites, } \\
\text { containing a total of } 4188 \text { sites. } \\
\text { bta-miR-2313-3p } \\
\text { CAGUUCC } \\
\text { CAGUUCU-bta-mir-3600 } \\
\text { CAAUUCC } \\
\text { CAAUUCU }\end{array}$ \\
& $\begin{array}{c}\text { bta-miR-2313-3p } \\
\text { CAGUUCC }\end{array}$ \\
& CAGUUCU \\
& bta-mir-3600 \\
& 3789 transcripts with \\
& predicted miRNA sites, \\
& containg a total of 4901 sites \\
\hline
\end{tabular}

